\title{
Comment on a Paper by Oyama and Shiokawa
}

\author{
Akihiro ABE \\ Department of Polymer Chemistry, Tokyo Institute of Technology, \\ Meguro-ku, Tokyo 152, Japan.
}

(Received January 13, 1982)

\begin{abstract}
KEY WORDS Conformational Energy / Polyethylene / Bond Conformation / Unperturbed Dimension / Integral Equation / Rotational Isomeric State Scheme /
\end{abstract}

Oyama and Shiokawa ${ }^{1}$ recently published a note showing the calculations of bond conformation and unperturbed dimension for the polyethylene (PE) chain by using a direct integration method. They adopted the formulation prescribed by Saito ${ }^{2}$ some years ago. The eigenvalues and eigenfunctions of the intergral equation whose kernel corresponds to the statistical weight defined for a pair of neighboring bond rotations, were evaluated numerically by using a potential energy contour map. The results were compared with those derived from calculations based on the rotational isomeric state (RIS) approximation in which statistical weights are expressed by simple Boltzmann factors in the corresponding conformational energies. Satisfactory agreement was found for the unperturbed mean-square end-toend distance $\left\langle r^{2}\right\rangle_{0}$. For the bond conformation, however, a somewhat higher estimate for the trans conformation was obtained when the RIS scheme was employed. These authors concluded from these results that the RIS scheme may be applicable for calculating $\left\langle r^{2}\right\rangle_{0}$, but not bond conformation. They suggested that such a discrepancy may arise from the fact that, in the RIS scheme adopted in their treatment, no consideration was made of the shape of the potential energy surface.

In this note, I would like to point out that a more elaborate RIS scheme ${ }^{3}$ has been available, allowing the shape of the potential energy minima to be taken into account. According to the procedure developed by Suter and Flory, ${ }^{3}$ the conformational partition function $z_{\zeta}$, average energy $\langle E\rangle_{\zeta}$, and averaged rotation angle $\left\langle\phi_{\mathrm{i}}\right\rangle_{\zeta}$ for each rotational state $\zeta$ may be calculated by

$$
\begin{gathered}
z_{\zeta}=\int_{\zeta} \int \exp (-E / R T) \mathrm{d} \phi_{\mathrm{i}} \mathrm{d} \phi_{\mathrm{i}+1} \\
\langle E\rangle_{\zeta}=z_{\zeta}{ }^{-1} \int_{\zeta} \int E \exp (-E / R T) \mathrm{d} \phi_{\mathrm{i}} \mathrm{d} \phi_{\mathrm{i}+1} \\
\left\langle\phi_{\mathrm{i}}\right\rangle_{\zeta}=z_{\zeta}{ }^{-1} \int_{\zeta} \int_{\mathrm{i}} \exp (-E / R T) \mathrm{d} \phi_{\mathrm{i}} \mathrm{d} \phi_{\mathrm{i}+1}
\end{gathered}
$$

where $\phi_{\mathrm{i}}$ and $\phi_{\mathrm{i}+1}$ represent the rotation angles for a pair of consecutive skeletal bonds $i$ and $i+1$, respectively. In the following treatment, the energies $E\left(\phi_{\mathrm{i}}, \phi_{\mathrm{i}+1}\right)$ were taken from the conformational energy contour map given by Oyama and Shiokawa. ${ }^{1}$ Boltzmann factors were calculated for each discrete set of $\phi_{\mathrm{i}}$ and $\phi_{\mathrm{i}+1}$ defined at $10^{\circ}$ intervals and integrations were carried out according to the trapezoidal rule over the range $E \leqq 5 \mathrm{kcal}$ $\mathrm{mol}^{-1}$ as prescribed by the energy contour map. The partition functions $z$, average energies $\langle E\rangle$, and averaged angles $\left\langle\phi_{\mathrm{i}}\right\rangle$ and $\left\langle\phi_{\mathrm{i}+1}\right\rangle$ calculated at three temperatures for the four nonequivalent states are listed in Table I. The energies are expressed relative to $E$ at the minimum for the $t t$ state, and correspondingly the partition functions are given relative to a value of unity for this state. The averaged rotation angles are nearly independent of temperature.

Oyama and Shiokawa ${ }^{1}$ used a $3 \times 3$ statistical weight matrix in their RIS treatment of the PE chain, which may be expressed customarily ${ }^{4}$ as

$$
\boldsymbol{U}=\left[\begin{array}{ccc}
1 & \sigma & \sigma \\
1 & \sigma & \sigma \omega \\
1 & \sigma \omega & \sigma
\end{array}\right]
$$




\section{A. ABE}

Table I. Partition functions, average energies, and averaged rotation angles deduced from the potential energy contour map ${ }^{a}$

\begin{tabular}{|c|c|c|c|c|c|c|c|c|c|}
\hline \multirow{2}{*}{$\begin{array}{l}\text { Rotational } \\
\text { state }\end{array}$} & \multicolumn{3}{|c|}{$z^{\mathrm{b}}$} & \multicolumn{3}{|c|}{$\langle E\rangle^{\mathrm{c}} / \mathrm{kcal} \mathrm{mol}^{-1}$} & \multicolumn{3}{|c|}{$\left\langle\phi_{\mathrm{i}}\right\rangle,\left\langle\phi_{\mathrm{i}+1}\right\rangle^{\mathrm{d}} / \mathrm{deg}$} \\
\hline & $300 \mathrm{~K}$ & $400 \mathrm{~K}$ & $500 \mathrm{~K}$ & $300 \mathrm{~K}$ & $400 \mathrm{~K}$ & $500 \mathrm{~K}$ & $300 \mathrm{~K}$ & $400 \mathrm{~K}$ & $500 \mathrm{~K}$ \\
\hline$t t$ & 1.000 & 1.000 & 1.000 & 0.64 & 0.85 & 1.04 & $0.0,0.0$ & $0.0,0.0$ & $0.0,0.0$ \\
\hline $\operatorname{tg}^{+}$ & 0.340 & 0.430 & 0.495 & 1.21 & 1.42 & 1.60 & $0.8,116.6$ & $0.9,116.4$ & $0.9,116.2$ \\
\hline$g^{+} g^{+}$ & 0.125 & 0.201 & 0.265 & 1.78 & 1.97 & 2.13 & $116.5,117.0$ & $116.4,116.9$ & $116.3,116.7$ \\
\hline$g^{+} g^{-}$ & 0.005 & 0.018 & 0.038 & 3.86 & 3.93 & 3.98 & $108.9,-108.9$ & $109.0,-109.0$ & $109.1,-109.1$ \\
\hline
\end{tabular}

a $c f$. Figure 1 of ref 1.

b Expressed relative to a value of unity for the $t t$ state.

c Energies expressed relative to zero for the minimum in the $t t$ state.

d Rotation angles are measured from the trans state $\left(\phi_{t}=0\right)$.

Table II. Conformational energies and preexponential factors associated with statistical weights

\begin{tabular}{crrr}
\hline & $300 \mathrm{~K}$ & $400 \mathrm{~K}$ & $500 \mathrm{~K}$ \\
\hline$E_{\sigma} / \mathrm{cal} \mathrm{mol}^{-1}$ & 570 & 560 & 550 \\
$E_{\omega} / \mathrm{cal} \mathrm{mol}^{-1}$ & 2080 & 1960 & 1840 \\
$\sigma_{0}$ & 0.92 & 0.91 & 0.89 \\
$\omega_{0}$ & 1.36 & 1.12 & 0.97 \\
\hline
\end{tabular}

where the elements are indexed in the order $t, g^{+}$, $g^{-}$. In this study, the statistical weight parameters $\sigma$ and $\omega$ are defined in terms of the conformational energies $E_{\sigma}$ and $E_{\omega}$, respectively:

$$
\begin{aligned}
& \sigma=\sigma_{0} \exp \left(-E_{\sigma} / R T\right) \\
& \omega=\omega_{0} \exp \left(-E_{\omega} / R T\right)
\end{aligned}
$$

The preexponential factors $\sigma_{0}$ and $\omega_{0}$ represent the effective sizes of the associated potential energy minima. Values of $E_{\sigma}, E_{\omega}, \sigma_{0}$, and $\omega_{0}$ were estimated from $\langle E\rangle$ and $z$ given in Table I according to the procedure set forth by Suter and Flory. ${ }^{3}$ The results are summarized in Table II. As shown on the energy contour map ${ }^{1}$ the shapes of the potential energy minima for the $t t, t g^{ \pm}$, and $g^{ \pm} g^{ \pm}$states are quite similar to each other except for the altitude of these minima. Correspondingly, the mean value of the preexponential factor $\sigma_{0}$ deduced by the analysis of these states is close to unity and stays nearly invariable over a wide range of temperature. The $g^{ \pm} g^{\mp}$ state involves severe steric interactions, and the energy contour diagram calculated for this region consists of a doublet. ${ }^{1}$ Values of $\left\langle\phi_{\mathrm{i}}\right\rangle$ and $\left\langle\phi_{\mathrm{i}+1}\right\rangle$ obtained by taking averages over the entire domain are given in Table I. The corresponding energy $\langle E\rangle$, expressed relative to the $t t$ state, varies from 3.2 to $2.9 \mathrm{kcal} \mathrm{mol}^{-1}$ in the range 300 to $500 \mathrm{~K}$ : the magnitude of $\langle E\rangle$ thus found is close to the minimum value $\left(E_{\min }=3.3 \mathrm{kcal} \mathrm{mol}^{-1}\right)$ reported for this state. ${ }^{1}$ The factor $\omega_{0}$ decreases from $1.3_{6}$ to $0.9_{7}$ over the same temperature range. From these results, we adopt the following expressions for the temperature of $140^{\circ} \mathrm{C}$ :

$$
\begin{aligned}
& \sigma=0.9 \exp (-560 / R T) \\
& \omega=1.1 \exp (-1940 / R T)
\end{aligned}
$$

with $R T$ expressed in cal mol${ }^{-1}$. Values of $\sigma_{0}$ and $\omega_{0}$ depart from unity only by $c a$. $10 \%$. This suggests that the effect arising from the dissimilarity of the shapes of the potential energy minima should be comparatively small in this polymer system.

In accordance with the treatment of Oyama and Shiokawa, ${ }^{1}$ we assume an infinitely long chain $(n=\infty)$ for which the largest-eigenvalue method can be applied. The largest eigenvalue of the matrix given in eq 4 takes the form ${ }^{4}$

$$
\lambda=(1 / 2)\left\{1+\sigma+\omega+\sqrt{[1-\sigma(1+\omega)]^{2}+8 \sigma}\right\}
$$

Bond conformations $\left(e . g ., f_{\xi \eta}\right)$ may be estimated for a consecutive pair of bonds according to the expression,

$$
f_{\xi \eta}=\partial \ln \lambda / \partial \ln u_{\xi \eta}
$$

where $u_{\xi \eta}$ denotes the $(\xi, \eta)$ element of $\boldsymbol{U}$ in eq 4 . Values of $f$ calculated for the $t t, t g^{ \pm}\left(g^{ \pm} t\right), g^{ \pm} g^{ \pm}$, and $g^{ \pm} g^{\mp}$ conformations are listed in Table III. These results are in good agreement with those 
Table III. Comparison of bond conformations estimated by various methods for the temperature of $140^{\circ} \mathrm{C}$

\begin{tabular}{|c|c|c|c|c|}
\hline \multirow[b]{2}{*}{$\begin{array}{c}\text { Bond } \\
\text { conformation }\end{array}$} & \multicolumn{3}{|c|}{ RIS approximation } & \multirow[b]{2}{*}{$\begin{array}{l}\text { Integration } \\
\text { method }^{\mathrm{a}}\end{array}$} \\
\hline & $\begin{array}{l}\text { Present } \\
\text { method } \\
(\text { eq 6) }\end{array}$ & $\begin{array}{l}\text { Minimum } \\
\text { energies } \\
\text { (eq 9) }\end{array}$ & $\begin{array}{c}\text { Mean } \\
\text { energies } \\
\text { (eq } 10)\end{array}$ & \\
\hline$f_{t}^{\mathbf{b}}$ & 0.626 & 0.609 & 0.605 & 0.618 \\
\hline$f_{g}{ }^{\mathrm{b}}$ & 0.374 & 0.391 & 0.395 & 0.382 \\
\hline$f_{t t}$ & 0.361 & 0.337 & 0.332 & 0.351 \\
\hline$f_{t g} \pm+f_{g \pm t}$ & 0.531 & 0.545 & 0.546 & 0.534 \\
\hline$f_{g^{ \pm} g^{ \pm}}$ & 0.098 & 0.110 & 0.112 & 0.101 \\
\hline$f_{g \pm g} \mp$ & 0.010 & 0.008 & 0.009 & 0.012 \\
\hline
\end{tabular}

a $c f$. Table I of ref 1 .

b Values of $\langle\cos \phi\rangle\left(=f_{t} \cos \phi_{t}+f_{g} \cos \phi_{g}\right)$ were calculated to be $0.459,0.435$, and 0.429 for eq.6, 9 , and 10 , respectively. The corresponding value deduced from the integration method $^{1}$ is 0.488 .

estimated from the direct integration method by Oyama and Shiokawa. ${ }^{1}$ Fractions of the conformer for a single bond, $f_{t}$ and $f_{g}$, can be deduced therefrom, and are included in the table. Also given in the table are the results obtained alternatively by adopting simpler expressions for the statistical weight parameters in eq 4 ; i.e., with the minimum energies, ${ }^{1}$

$$
\begin{aligned}
& \sigma=\exp (-550 / R T) \\
& \omega=\exp (-2200 / R T)
\end{aligned}
$$

and with the energies termed "mean" values, ${ }^{1}$

$$
\begin{aligned}
& \sigma=\exp (-540 / R T) \\
& \omega=\exp (-2030 / R T)
\end{aligned}
$$

As shown in the table, use of these alternative parameter sets yields very similar results with those derived from the more elaborate methods: e.g., values of $f_{t}\left(=1-f_{g}\right)$ calculated as above all fall in the range $0.615 \pm 0.011$. In this respect, the present results are at variance with those of Oyama and Shiokawa, ${ }^{1}$ who obtained a somewhat higher content for the trans conformation $\left(f_{t}=0.721 \pm 0.003\right)$ when a parameter set such as those given in eq 9 and 10 was employed. Since the details of the computational procedure have not been reported, ${ }^{1}$ the cause for this discrepancy can not be identified.

The characteristic ratio $C_{\infty}$ for the unperturbed end-to-end distance and its temperature coefficient $\mathrm{d} \ln C_{\infty} / \mathrm{d} T$ were also calculated by using the parameter set given in eq 6 . When geometrical pa- rameters such as $\angle C C C=112^{\circ}, \phi_{t}=0$, and $\phi_{g^{ \pm}}=$ $\pm 116.5^{\circ}$ were used, the following values calculated ${ }^{4}$ for an infinitely long PE chain were obtained: $C_{\infty}=$ 7.84 and $\mathrm{d} \ln C_{\infty} / \mathrm{d} T=-1.23 \times 10^{-3} \mathrm{~K}^{-1}$ at $140^{\circ} \mathrm{C}$. These results ${ }^{5}$ should be compared with those obtained by adopting the energy parameters given by Oyama and Shiokawa. ${ }^{1}$ Use of the parameter set for the minimum energy (eq 9) yielded $C_{\infty}=7.65$ and $\mathrm{d} \ln C_{\infty} / \mathrm{d} T=-1.06 \times 10^{-3} \mathrm{~K}^{-1}\left(140^{\circ} \mathrm{C}\right)$. The parameter set for the mean energy (eq 10) gave $C_{\infty}=$ 7.51 and $\mathrm{d} \ln C_{\infty} / \mathrm{d} T=-1.10 \times 10^{-3} \mathrm{~K}^{-1}\left(140^{\circ} \mathrm{C}\right)$. In these calculations, geometrical parameters were taken to be identical with those given above. Variation arising from the choice of the statistical weight parameter set is quite insignificant for either $C_{\infty}$ or $\mathrm{d} \ln C_{\infty} / \mathrm{d} T$.

In summary, I would like to call attention to the fact that, in the RIS scheme developed by Flory, ${ }^{3,4}$ due consideration is given to the effect arising from the shape of the potential energy minima. The calculations presented in this note indicate, however, that the simple RIS treatment gives a satisfactory presentation of the bond conformation of the PE chain. This conclusion is at variance with Oyama and Shiokawa. ${ }^{1}$ Allegra and Immirzi ${ }^{6}$ examined the Fourier series development of the statistical weight function, and concluded that their method should be preferable to the RIS treatment in such a case that the energy minima are flat and/or the energy barriers are low compared to $R T$. The same seems to apply to the integration method developed by Oyama and Shiokawa. ${ }^{1}$ 


\section{REFERENCES}

1. T. Oyama and K. Shiokawa, Polym. J., 13, 1145 (1981).

2. N. Saito in "Kobunshi no Butsuri," Physical Society of Japan, Ed., Asakura, Tokyo, 1963; N. Saito, K. Okano, S. Iwayanagi, and T. Hideshima, Solid State Phys., 14, 344 (1963).

3. U. W. Suter and P. J. Flory, Macromolecules, 8, 765
(1975).

4. P. J. Flory, "Statistical Mechanics of Chain Molecules," Interscience, New York, N.Y., 1969.

5. These calculations follow directly from calculated conformational energies. It is for this reason that characteristic ratios exceed the experimental values somewhat.

6. G. Allegra and A. Immirzi, Makromol. Chem., 124, 70 (1969). 